\title{
ARTICLE
}

\section{Low serum osteocalcin levels are correlated with left ventricular systolic dysfunction and cardiac death in Chinese men}

\author{
Xue-li Zhang ${ }^{1}$, Yun Shen ${ }^{1}$, Xiao-jing Ma ${ }^{1}$, Zhi-gang $\mathrm{Lu}^{2}$, Yi-ting $\mathrm{Xu}^{1}$, Qin Xiong ${ }^{1}$ and Yu-qian Bao ${ }^{1}$
}

\begin{abstract}
Osteocalcin is a newly identified type of cytokine secreted by osteoblasts, which has an endocrine function, mediates energy and glycol-lipid metabolism, and is closely related to cardiovascular diseases. In this study, we investigated the value of serum osteocalcin levels in predicting left ventricular systolic dysfunction and cardiac death. A total of 258 patients in the Department of Cardiology were included. Two-dimensional echocardiography was performed in all the subjects. The cardiac death of subjects occurring with a median follow-up of 4.6 years was informed via phone calls or the electronic medical records. The serum osteocalcin levels were measured using electrochemiluminescent immunoassay. We found that the median left ventricular ejection fractions (LVEFs) were $62 \%$ in men and $63 \%$ in women. In the men with a LVEF $>62 \%$, the serum osteocalcin levels were significantly higher than in those with LVEF $\leq 62 \%(P=0.042)$, whereas this difference was absent in the women. Both the serum osteocalcin $(\beta=0.095, P=0.028)$ and serum $N$-terminal pro-brain natriuretic peptide (NT-pro-BNP; $\beta=-0.003, P<0.01)$ levels remained independently significantly correlated with LVEF in the men but not in the women. Receiver operating characteristic (ROC) analyses of the men revealed that the serum osteocalcin $(P=0.007)$, serum NT-pro-BNP $(P=0.018)$ and serum osteocalcin + NT-pro-BNP $(P<0.01)$ levels were all significant in identifying left ventricular systolic dysfunction at baseline, but the pairwise comparisons of the three areas under the curves (AUCs) were all non-significant. The men in the lower osteocalcin level group at baseline suffered a greater risk of future cardiac death than those in the higher osteocalcin level group, whereas the result for NT-pro-BNP exhibited the opposite pattern. In conclusion, lower serum osteocalcin levels in the men could identify left ventricular systolic dysfunction and cardiac death in a manner that was not inferior to high serum NT-pro-BNP levels.
\end{abstract}

keywords: cardiac death; coronary artery disease; left ventricular systolic dysfunction; osteocalcin; N-terminal pro-brain natriuretic peptide

Acta Pharmacologica Sinica (2019) 40:486-491; https://doi.org/10.1038/s41401-018-0080-0

\section{INTRODUCTION}

Previous studies have revealed that bone metabolic diseases are closely correlated with cardiovascular diseases [1]. Patients with cardiovascular diseases often have metabolic bone diseases [2]. In recent years, the incidences of both types of disease mentioned above have gradually increased with the aging of the population, which has influenced the longevity and quality of life to a great extent. Previous studies have demonstrated that bone metabolic diseases and cardiovascular diseases have some common pathophysiological mechanism and risk factors, for example, aging, menopause, smoking, a sedentary lifestyle, and inflammation [3, 4]. Osteocalcin is secreted by osteoblasts and is a newly identified type of cytokine. Osteocalcin has an endocrine function, mediates energy and glycol-lipid metabolism, and is closely related to cardiovascular diseases [5]. Studies have demonstrated that serum osteocalcin levels are negatively correlated with the extent of vasculopathy in coronary artery diseases (CAD), i.e., with increases in lesion vessel numbers, the serum osteocalcin levels decrease [6]. Serum osteocalcin levels are closely related to the prognosis of $C A D$ and act as an important hemodynamic parameter for assessing this prognosis. In chronic heart failure patients with low left ventricular ejection fractions (LVEFs), the incidence of fractures significantly increases with decreases in serum osteocalcin levels [7]. In our previous study that utilized a mouse model, we found that an intervention of exogenous osteocalcin significantly attenuated the status of atherosclerosis [8]. Therefore, this study aimed to investigate the relationship between serum osteocalcin levels and the indexes of cardiac function and cardiac death.

\section{MATERIALS AND METHODS}

Subjects

A total of 276 patients from the Department of Cardiology of Shanghai Jiao Tong University Affiliated Sixth People's Hospital

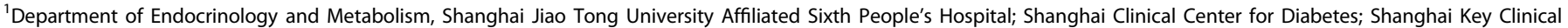

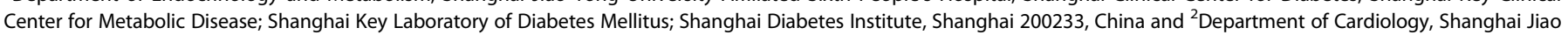
Tong University Affiliated Sixth People's Hospital, Shanghai 200233, China

Correspondence: Yu-qian Bao (yqbao@sjtu.edu.cn)

These authors contributed equally: Xue-li Zhang, Yun Shen.

Received: 7 March 2018 Accepted: 18 June 2018

Published online: 10 July 2018 
from January 2008 to December 2009 were included in this study. All of the subjects underwent diagnostic coronary angiography and echocardiography during hospitalization. This study was approved by the local Ethics Committee of Shanghai Jiao Tong University Affiliated Sixth People's Hospital, and all subjects provided informed consent. The subjects were followed up from March 2015 to August 2016 by phone calls or through the electronic medical records. The primary follow-up outcome was cardiac death. The exclusion criteria were as follows: (1) severe valvular heart disease or atrial fibrillation; (2) inflammation, fracture, malignant tumor, liver or renal failure; (3) treatment with medicines that may affect bone metabolism such as bisphosphonates, corticosteroids or warfarin; and (4) insufficient laboratory or echocardiographic data. Overall, 258 patients were included in the final analysis, and the median follow-up duration was 4.6 years.

\section{Diagnostic criteria}

Coronary angiography was conducted using the standard Judkins technique, and CAD was diagnosed when stenosis of $\geq 50 \%$ of the lumen diameter was detected in a major coronary artery, including the left main coronary artery, left anterior descending artery or its first diagonal branch, left circumflex artery or its first obtuse marginal branch, and the right coronary artery [9].

Diabetes was defined according to the World Health Organization (WHO) 1999 criteria as a fasting plasma glucose (FPG) of at least $7.0 \mathrm{mmol} / \mathrm{L}$ and/or a 2-h postprandial plasma glucose (2 hPG) measurement of at least $11.1 \mathrm{mmol} / \mathrm{L}$ or a previous diagnosis of diabetes [10].

Hypertension was defined according to the WHO/International Society of Hypertension (ISH) 2003 criteria as a systolic blood pressure (SBP) $\geq 140 \mathrm{~mm} \mathrm{Hg}$ or a diastolic blood pressure (DBP) $\geq 90 \mathrm{~mm} \mathrm{Hg}$ or having undergone a specific treatment for hypertension [11].

The subjects were identified as "current smokers" if they reported regular use of inhaled tobacco products or had smoked at least one cigarette per day at any time during the previous 6 months [12].

Graded left ventricular dysfunction was defined according to the recommendations for cardiac chamber quantification by echocardiography in adults published by the American Society of Echocardiography and the European Association of Cardiovascular Imaging as a LVEF in the normal range $(52-72 \%$ for men, $54-74 \%$ for women), in the mildly abnormal range $(41-51 \%$ for men, $41-53 \%$ for women), in the moderately abnormal range $(30-40 \%)$ or in the severely abnormal range $(<30 \%)$ [13].

Anthropometric and laboratory measurements

Body mass index (BMI) was calculated as the weight divided by the squared height. The waist circumference was measured midway between the lowest rib and the superior border of iliac crest on the midaxillary line.

Blood samples were taken from all of the study participants after an overnight fast $(\geq 10 \mathrm{~h})$ and stored at $-80^{\circ} \mathrm{C}$ until testing. FPG and 2 hPG were determined by the glucose oxidase method. Fasting insulin was assayed with a radioimmunoassay (Linco Research, St Charles, MO, USA). Insulin resistance was estimated via the homeostasis model assessment index (HOMA-IR) as follows: HOMA-IR $=$ FPG $(\mathrm{mmol} / \mathrm{L}) \times$ fasting serum insulin $(\mathrm{mU} / \mathrm{L}) / 22.5$. The glycated hemoglobin $\mathrm{A} 1 \mathrm{c}(\mathrm{HbA} 1 \mathrm{c})$ concentration was measured via high-pressure liquid chromatography (Bio-Rad Inc, Hercules, CA, USA). Triglycerides (TG), highdensity lipoprotein cholesterol (HDL-c), and low-density lipoprotein cholesterol (LDL-c) were determined by standard enzymatic procedures on an automated bioanalyzer (7600-020; Hitachi, Tokyo, Japan). C-reactive protein (CRP) was measured with a particle-enhanced immunonephelometry assay (Dade Behring Inc, Newark, NJ, USA).
The serum NT-pro-BNP levels were assayed by chemiluminescence (Cobas 6000, Roche Diagnostics GmbH, Mannheim, Germany), and the inter-assay and intra-assay coefficients of variation were $4.6 \%$ and $4.2 \%$, respectively. The serum total osteocalcin levels were quantified via electrochemiluminescent immunoassay (Roche Diagnostics, GmbH, Mannheim, Baden-Württemberg, Germany) on a Roche Elecsys 2010 (Elecsys module) immunoassay analyzer. This total osteocalcin assay detects the major N-terminal fragment. The inter- and intraassay coefficients of variation were $1.7-6.5 \%$ and $1.2-4.0 \%$, respectively.

\section{Echocardiography}

Echocardiography was performed in all subjects using a Philips IE33 ultrasound system with a $2.5-\mathrm{MHz}$ transducer. Echocardiographic determination of the left atrial dimension (LAD), left ventricular end-diastolic dimension (LVDd), left ventricular end systolic dimension (LVSd), the interventricular septum dimension (IVST), the left ventricular posterior wall thickness (PWT), left ventricular ejection fraction (LVEF), and fractional shortening (FS) were recorded and evaluated by an experienced echocardiographer. The left ventricular mass (LVM) was calculated with a formula from Devereux et al. as $L V M=0.8 \times$ $1.04 \times\left[(\text { LVDd + IVST + PWT })^{3}-\right.$ LVDd $\left.^{3}\right]+0.6$. The LVM index (LVMI) was calculated as the LVM divided by the body surface area [14].

Statistical analysis

All of the statistical analyses were performed with SPSS version 21.0 (SPSS, Chicago, IL, USA). The one-sample KolmogorovSmirnov test was used to explore the normalities of the distributions of the data. Normally distributed data are expressed in terms of the means \pm the standard deviations, and the skewed data are expressed in terms of the medians (with the inter-quartile ranges). The clinical data were compared between the two groups using unpaired Student's $t$ tests (for normally distributed data) or Mann-Whitney $U$ tests (for the skewed data). The $X^{2}$ test was used to compare the categorical variables. The serum cutoff values and their corresponding sensitivities and specificities for osteocalcin, NT-pro-BNP and osteocalcin + NT-pro-BNP for the identification of left ventricular systolic dysfunction at baseline were analyzed by receiver operating characteristic curve (ROC) analyses [15]. The areas under the curves (AUCs) were compared by pairwise comparison analysis. Survival curves were analyzed by performing log-rank tests with Kaplan-Meier figures. Two-tailed $P$ values $<0.05$ were considered statistically significant.

\section{RESULTS}

Baseline characteristics

This study included 158 men $(65.03 \pm 10.46$ years old $)$ and 100 women (67.68 \pm 9.17 years old). All of the women were postmenopausal. The serum osteocalcin levels were significantly higher in the women than in the men $[23.80 \mathrm{ng} / \mathrm{mL}(17.97-32.93)$ vs. $17.12 \mathrm{ng} / \mathrm{mL}(13.29-23.72)$, respectively, $P<0.01]$, and the serum NT-pro-BNP levels were also much higher in the women than in the men $[140.70 \mathrm{ng} / \mathrm{L}(67.00-318.73)$ vs. $96.78 \mathrm{ng} / \mathrm{L}$ (39.75-277.03), respectively, $P=0.039]$. We further separated the men and women according to the median LVEF $(62 \%$ for men and $63 \%$ for women). As presented in Table 1, we found that in both the men and women, age, BMI, blood pressure, plasma glucose, lipid profiles and the history of medicines did not differ significantly between the different LVEF groups, whereas the $\mathrm{HbA} 1 \mathrm{c}$ value did differ significantly $[6.2 \%(5.8-6.9 \%)$ vs. $6.0 \%(5.6-6.5 \%), P=0.045]$. In the men with a LVEF $>62 \%$, the serum osteocalcin levels were significantly higher than those in the men with a LVEF $\leq 62 \%(P=0.042)$, and the serum NT-pro-BNP 
Table 1. Characteristics of the study participants

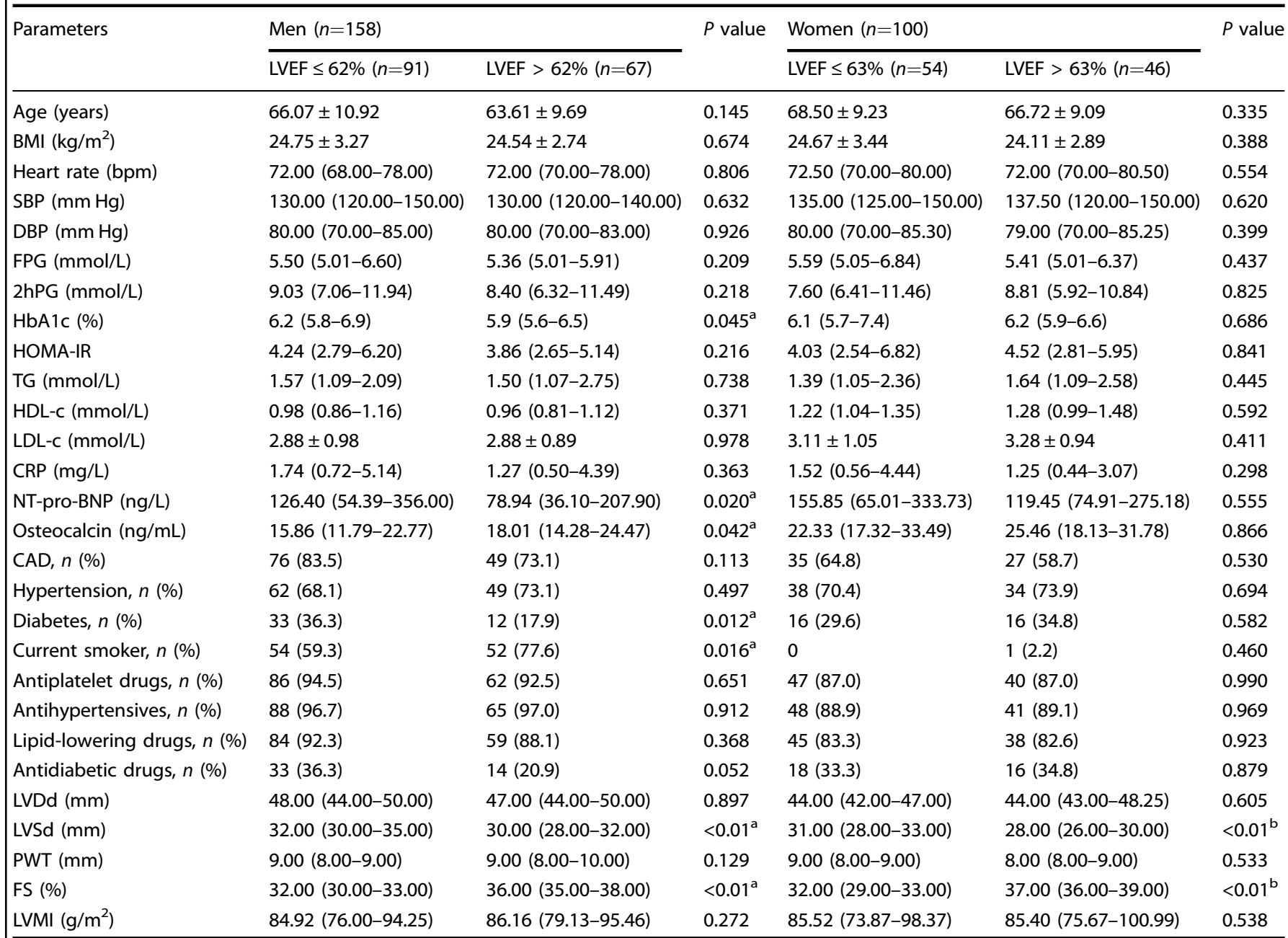

$A O$ aortic diameter, $B M I$ body mass index, $C A D$ coronary artery disease, $C R P$ C-reactive protein, $D B P$ diastolic blood pressure, $F P G$ fasting plasma glucose, FS fraction shortening, HbA1c glycated hemoglobin A1c, HDL-c high-density lipoprotein cholesterol, HOMA-IR Homeostasis model assessment-insulin resistance, IVST interventricular septal thickness, LAD left atrial diameter, LVDd left ventricular end-diastolic dimension, LVEF left ventricular ejection fraction, LVMI left ventricular mass index, LVSd left ventricular end-systolic dimension, LDL-c low-density lipoprotein cholesterol, NT-pro-BNP N-terminal pro-brain natriuretic peptide, PWT posterior wall thickness, SBP systolic blood pressure, TG Triglyceride, 2 hPG 2-h post plasma glucose

The data are means \pm s.d., median (interquartile range) or $n(\%)$

${ }^{\mathrm{a}} P<0.05$ vs. LVEF $\leq 62 \%$ in men

${ }^{\mathrm{b}} P<0.01$ vs. LVEF $\leq 63 \%$ in women

levels were much lower in the former group $(P=0.02)$. Regarding the echocardiographic parameters, the LVSd was much lower in the men with a LVEF $>62 \%$ than in those with a $\operatorname{LVEF} \leq 62 \%$, while the FS was much higher in the same comparison (all $P<0.01$ ). In the women, there were no significant differences between any of the variables with the exceptions of the LVSd and FS (all $P<0.01$ ). Considering these results in the women, we conducted the following analyses only in the men.

The independent relationship between serum osteocalcin level and left ventricular systolic function in men

To investigate the independent relationship between serum osteocalcin level and left ventricular systolic function in men, we conducted a stepwise regression analysis using LVEF as the independent variable. As indicated in Table 2, after including age, BMI, SBP, DBP, HOMA-IR, TG, HDL-C, LDL-C, $C R P$, osteocalcin, NT-pro-BNP, current smoking status, CAD,
Table 2. Multiple stepwise regression analysis showing variables independently associated with left ventricular systolic function in men

\begin{tabular}{lllll}
\hline Independent variables & $\beta$ & S.E. & Standardized $\beta$ & $P$ value \\
\hline Osteocalcin & 0.105 & 0.043 & 0.188 & 0.016 \\
NT-pro-BNP & -0.003 & 0.001 & -0.327 & $<0.01$ \\
\hline
\end{tabular}

Variables included in the original model are age, BMI, SBP, DBP, HOMA-IR, TG, HDL-c, LDL-c, CRP, osteocalcin, NT-pro-BNP, current smoker, CAD, antiplatelet drugs, antihypertensives, lipid-lowering drugs, antidiabetic drugs

*Only statistical significant factors are presented in Table 2 after stepwise regression analysis

antiplatelet drugs, antihypertensives, lipid-lowering drugs, and antidiabetic drugs into a regression model, only osteocalcin $(\beta=0.105, \quad P=0.016)$ and NT-pro-BNP $(\beta=-0.003, \quad P<0.01)$ remained significant. 
The relationships of serum osteocalcin, NT-pro-BNP and osteocalcin + NT-pro-BNP with left ventricular systolic dysfunction at baseline in men

We constructed curves for osteocalcin, NT-pro-BNP and osteocalcin + NT-pro-BNP to identify left ventricular systolic dysfunction in the men at baseline (defined as a LVEF $<52 \%$ ) via ROC analyses (Fig. 1). The optimal cutoff for osteocalcin for identifying left ventricular systolic dysfunction at baseline was $17.88 \mathrm{ng} / \mathrm{mL}$, and the corresponding sensitivity and specificity were $71.4 \%$ and $53.6 \%$, respectively. The AUC was 0.733 (95\% confidence interval $0.564-0.902, P=0.007)$. The optimal cutoff for NT-pro-BNP for identifying the left ventricular systolic dysfunction at baseline was $278.60 \mathrm{ng} / \mathrm{L}$ with a corresponding sensitivity of

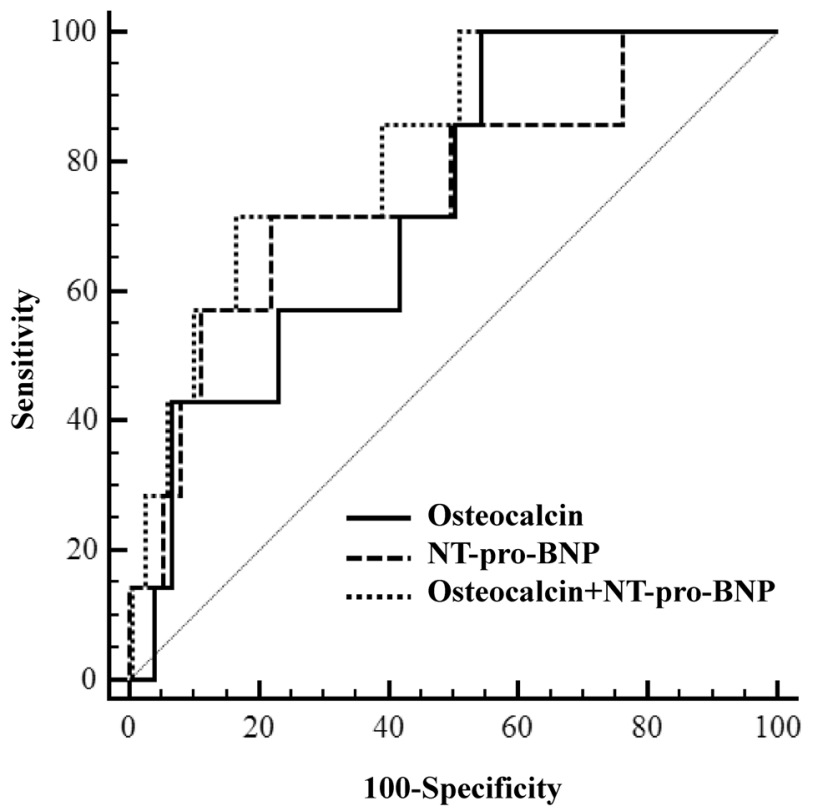

Fig. 1 ROC curves for identifying left ventricular systolic dysfunction and comparisons of the AUCs. Osteocalcin model, AUC $=0.733$ (95\% confidence interval 0.564-0.902); NT-pro-BNP model, AUC = 0.754 (95\% confidence interval 0.543-0.965); Osteocalcin + NT-proBNP model, AUC $=0.820$ (95\% confidence interval 0.671-0.969) Comparisons of the AUCs: Osteocalcin model vs. NT-pro-BNP model, $P=0.885$; osteocalcin model vs. osteocalcin + NT-pro-BNP model, $P=0.279 ; \quad N T-p r o-B N P$ model vs. osteocalcin + NT-proBNP model, $P=0.483$

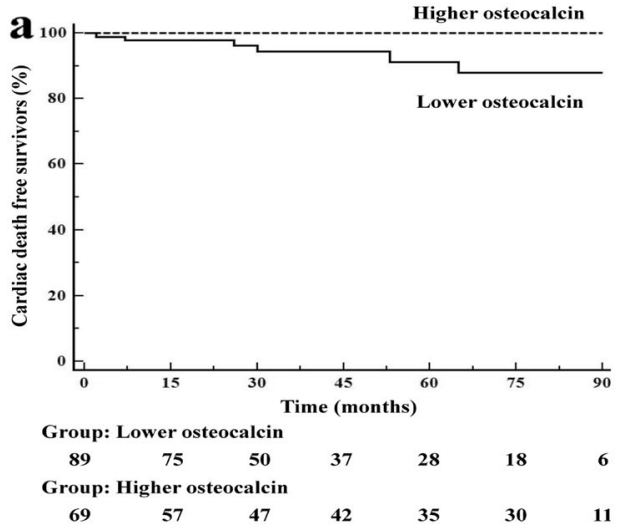

$71.4 \%$ and a specificity of $78.1 \%$. The AUC was $0.754(95 \%$ confidence interval $0.543-0.965, P=0.020)$. When osteocalcin and NT-pro-BNP were combined, the corresponding sensitivity was still $71.4 \%$, and the specificity increased from $53.6 \%$ to $83.4 \%$. The AUC was 0.820 (95\% confidence interval $0.671-0.969$ ). The pairwise comparisons among the three curves were all nonsignificant (osteocalcin vs. NT-pro-BNP, $P=0.885$; osteocalcin vs. osteocalcin + NT-pro-BNP, $P=0.279$; NT-pro-BNP vs. osteocalcin + NT-pro-BNP, $P=0.483$ ).

The relationships of serum osteocalcin levels and serum NT-pro-BNP levels with cardiac death

We recorded 6 cases of cardiac death due to heart failure in men with a median follow-up of 4.6 years. We used the optimal cutoffs for osteocalcin and NT-pro-BNP calculated above to separate the patients into a higher osteocalcin level group and a lower osteocalcin level group as well as a higher NT-pro-BNP level group and a lower NT-pro-BNP level group. Further Kaplan-Meier survival analyses were performed. As illustrated in Fig. 2, the patients in the lower osteocalcin level group at baseline suffered a greater risk of future cardiac death than those in the higher osteocalcin level group ( $P=0.016$, Fig. 2a). The result for NT-proBNP exhibited the opposite pattern. The patients in the higher NT-pro-BNP level group at baseline suffered a greater risk of future cardiac death than did those in the lower NT-pro-BNP level group ( $P=0.008$, Fig. $2 b)$.

\section{DISCUSSION}

The study demonstrates that as serum osteocalcin levels increase, the left ventricular ejection fraction can be maintained at a certain level without deteriorating, and the risk of cardiac death is significantly lower in men with higher serum osteocalcin levels than in those with lower serum osteocalcin levels. This study demonstrates that the serum osteocalcin level may be involved in ventricular remodeling and provides a new perspective about the pathophysiological mechanism of osteocalcin in the diagnosis of cardiovascular diseases. Compared with NT-pro-BNP, which is the traditional marker for assessing heart failure, serum osteocalcin levels were not inferior in the identification of cardiac function and cardiac death. Low serum osteocalcin levels were able to identify left ventricular systolic dysfunction at baseline and a high risk of cardiac death longitudinally.

Clinical evidence has demonstrated that serum osteocalcin levels are correlated with cardiac function and play a protective role. A study by Schleithoff et al. included 21 men with New York

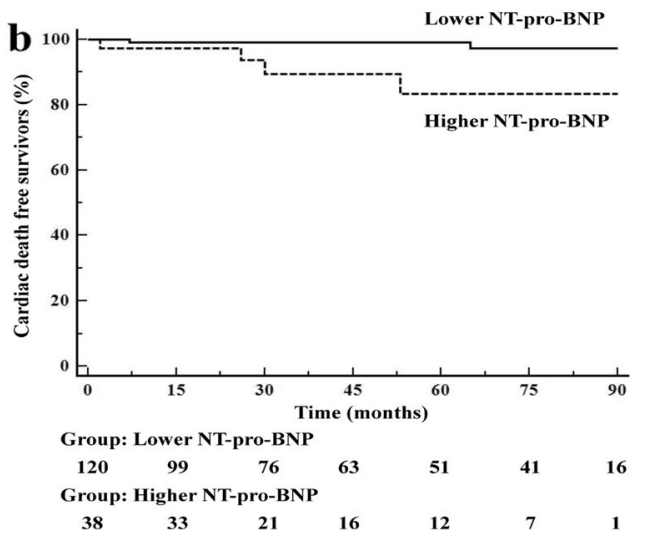

Fig. 2 Kaplan-Meier survival curves for cardiac death in the patients grouped according to the optimal cutoffs for serum osteocalcin and serum NT-pro-BNP levels in men. a The dashed line indicates the higher serum osteocalcin level group (>17.88 ng/mL), and the full line indicates the lower serum osteocalcin level group $(\leq 17.88 \mathrm{ng} / \mathrm{mL}, P=0.016)$. b The dashed line indicates the higher serum NT-pro-BNP level group ( $>278.60 \mathrm{ng} / \mathrm{L})$, and the full line indicates the lower serum NT-pro-BNP level group $(\leq 278.60 \mathrm{ng} / \mathrm{L}, P=0.008)$ 
Heart Association over grade II and 21 normal controls. These authors found that, in the patients with heart failure, the serum osteocalcin levels were relatively low [7]. Verheyen et al. found that indicators reflecting bone transformation were correlated with left ventricular function. After adjustment for other confounding factors, the serum osteocalcin levels still presented a positive correlation with mitral annulus velocity [16]. However, the conclusions about the relationship between serum osteocalcin levels and cardiac function are not consistent. In 73 patients with mild-to-moderate heart failure, Bozic et al. found that the serum osteocalcin levels were increased compared with those in normal controls after matching for the BMI [17]. Conversely, Wu et al. found that there was no significant difference between patients with heart failure and normal controls [18]. On the basis of a review of the literature, to our knowledge, we are the first to report the relationship between serum osteocalcin level and LVEF. In the present study, we found that lower serum osteocalcin levels were correlated with a higher risk of left ventricular systolic dysfunction, which provides novel evidence of a protective role of serum osteocalcin for cardiac function.

Yeap et al. found that total serum osteocalcin levels could predict both all-cause and cardiovascular disease mortality in men aged 70-89 years, but the osteocalcin levels presented as a U-shaped curve in this relationship [19]. Another study in Japan found that serum osteocalcin levels that were lower than the median value were associated with a higher risk of developing cardiovascular events in 126 hemodialysis patients after 5 years follow-up [20]. However, another study reported that serum osteocalcin levels might not be associated with the future risk of cardiovascular diseases [21]. Ethnic differences and different diagnostic criteria may have contributed to the diversity of the results to some extent. In our study, we found that serum osteocalcin levels could identify the risk of cardiac death within the following 4.6 years, which is convincing because this is a report of the association between serum osteocalcin levels and a hard end point.

Serum osteocalcin levels are negatively correlated with cardiovascular risk factors, such as obesity, diabetes, dyslipidemia and nonalcoholic fatty liver disease [6, 22, 23]. Experimental and clinical evidence has demonstrated that serum osteocalcin levels play a protective role in cardiovascular diseases [5]. Perturbed glycol-lipid metabolism is a well-known risk factor for cardiovascular diseases [24]. Serum osteocalcin may be involved in regulating energy metabolism and glycol-lipid metabolism through its binding with the $G$ protein-coupled receptor family C, group 6, member A (GPRC6A) receptor, which is regarded as a vascular-protective cytokine [25]. The GPRC6A receptor is a newly discovered $G$ protein-coupled receptor that is expressed in myocardial tissues [26]. Serum osteocalcin may play an important role in protecting myocardial cells by binding with the GPRC6A receptor. The mechanism of the protection against cardiomyopathy mediated by osteocalcin in mice with type 2 diabetes was studied by Gamal et al. [27]. These authors found that serum osteocalcin levels and serum adiponectin levels in the diabetes group were much lower than the levels in the control group. The expression of the GPRC6A receptor was also downregulated. However, in the control group that received a subcutaneous injection of warfarin, the serum osteocalcin levels and the serum adiponectin levels increased with the upregulation of the expression of the GPRC6A receptor. Serum osteocalcin could play a protective role in myocardial tissues by upregulating the levels of adiponectin, which could result in the upregulation of the expression of the GPRC6A receptor. Results from Gamal et al. also indicated that serum osteocalcin might exhibit a protective role via an anti-oxidative and anti-apoptotic mechanisms. In our previous study, we used apolipoprotein E-knockout mice to create a model of atherosclerosis. We found that an intervention with exogenous osteocalcin significantly attenuated high fat dietinduced impairment of endothelium-dependent relaxation through the phosphatidylinositol 3-kinase (PI3K)/protein kinase B (Akt)/endothelial nitric oxide synthase (eNOS) signaling pathway [8]. Jung et al. confirmed that osteocalcin can attenuate the impairment of human aortic endothelial cells caused by free fatty acid via the PI3K/Akt pathway [28].

Similarly, in our study, we also found that there was a significant difference in the serum osteocalcin levels between the men and women, which accords with previous findings [29]. The difference in the bone turnover rates between men and women, especially postmenopausal women, might primarily account for the gender difference in osteocalcin. Bone turnover rates are accelerated with increasing age, especially in postmenopausal women [30]. Among menopausal women, insufficient calcium and phosphorus may result in a low rate of the formation of hydroxyapatite crystals, which is regarded as a state of low bone mineralization. In this state, free osteocalcin may be secreted into the circulation, which would cause the increased concentration of osteocalcin in the sera of postmenopausal women, which in turn, may explain the reason for the gender difference, particularly in postmenopausal women [31].

There are some limitations to our study. First, this study was a retrospective study with a relatively small sample size. Second, all of the patients came from the department of cardiology, so this population was not generalized. Third, the patients were included in this study from 2008 to 2009; thus, recruitment began 10 years ago. Finally, we only measured the baseline serum osteocalcin levels and did not collect another measurement during the followup, so the dynamic changes in serum osteocalcin levels could not be observed.

\section{CONCLUSION}

We conclude that serum osteocalcin levels are significantly different between men and women. In men, lower serum osteocalcin levels are closely correlated with left ventricular function and can predict future cardiac death.

\section{ACKNOWLEDGEMENTS}

The authors thank all the staff of the Department of Cardiology, Shanghai Jiao Tong University Affiliated Sixth People's Hospital for their efforts in this study. This study was supported by the Translational Medicine Innovation Foundation of Shanghai Jiao Tong University School of Medicine (15ZH4006), and National Natural Science Foundation of China (31571212).

\section{AUTHOR CONTRIBUTION:}

$\mathrm{X}-\mathrm{JM}$ and $\mathrm{Y}-\mathrm{QB}$ designed the study and revised the manuscript; Y-TX, QX, X-LZ and YS performed the data collection; $X-L Z$ and $Y S$ performed the analyses and drafted the manuscript; Z-GL performed the angiography at baseline. All authors have revised the paper critically for intellectual content and approved the final version.

\section{ADDITIONAL INFORMATION}

Competing interests: The authors declare no competing interests.

\section{REFERENCES}

1. Sennerby U, Melhus H, Gedeborg R, Byberg L, Garmo H, Ahlbom A, et al. Cardiovascular diseases and risk of hip fracture. JAMA. 2009;302:1666-73.

2. Lai SW, Liao KF, Lai HC, Tsai PY, Lin $\mathrm{CL}_{\text {, }}$ Chen $\mathrm{PC}$, et al. Risk of major osteoporotic fracture after cardiovascular disease: a population-based cohort study in Taiwan. J Epidemiol. 2013;23:109-14.

3. Reyes-Garcia R, Rozas-Moreno P, Jimenez-Moleon JJ, Villoslada MJ, GarciaSalcedo JA, Santana-Morales $S$, et al. Relationship between serum levels of osteocalcin and atherosclerotic disease in type 2 diabetes. Diabetes Metab. 2012;38:76-81. 
4. Szulc P, Garnero P, Claustrat B, Marchand F, Duboeuf F, Delmas PD. Increased bone resorption in moderate smokers with low body weight: the Minos study. J Clin Endocrinol Metab. 2002;87:666-74.

5. Lee NK, Sowa $H$, Hinoi $E$, Ferron $M$, Ahn JD, Confavreux $C$, et al. Endocrine regulation of energy metabolism by the skeleton. Cell. 2007;130:456-69.

6. Bao YQ, Zhou M, Lu ZG, Li HT, Wang Y, Sun LQ, et al. Serum levels of osteocalcin are inversely associated with the metabolic syndrome and the severity of coronary artery disease in Chinese men. Clin Endocrinol (Oxf). 2011;75:196-201.

7. Schleithoff SS, Zittermann A, Stüttgen B, Tenderich G, Berthold HK, Körfer R, et al. Low serum levels of intact osteocalcin in patients with congestive heart failure. Jone Miner Metab. 2003;21:247-52.

8. Dou JX, Li HT, Ma XJ, Zhang ML, Fang QC, Nie MY, et al. Osteocalcin attenuates high fat diet-induced impairment of endothelium-dependent relaxation through Akt/eNOS-dependent pathway. Cardiovasc Diabetol. 2014;13:74.

9. Judkins MP. Percutaneous transfemoral selective coronary arteriography. Radiol Clin North Am. 1968;6:467-92.

10. World Health Organization. Definition, diagnosis and classification of diabetes mellitus and its complications: report of a WHO consultation. Part 1: diagnosis and classification of diabetes mellitus. WHO, Geneva, Switzerland 1999.

11. Whitworth JA, World Health Organization. International Society of Hypertension Writing Group. World Health Organization (WHO)/International Society of Hypertension (ISH) statement on management of hypertension. J Hypertens. 2003;21:1983-92.

12. Yang GH, Fan LX, Tan J, Qi GM, Zhang YF, Samet JM, et al. Smoking in China: findings of the 1996 National Prevalence Survey. JAMA. 1999;282:1247-53.

13. Lang RM, Badano LP, Mor-Avi V, Afilalo J, Armstrong A, Ernande L, et al. Recommendations for cardiac chamber quantification by echocardiography in adults: an update from the American Society of Echocardiography and the European Association of Cardiovascular Imaging. Eur Heart J Cardiovasc Imaging. 2015;16:233-70.

14. Devereux RB, Reichek N. Echocardiographic determination of left ventricular mass in man. Anat Valid Method Circ. 1977;55:613-8.

15. Zou $\mathrm{KH}$, O'Malley AJ, Mauri L. Receiver-operating characteristic analysis for evaluating diagnostic tests and predictive models. Circulation. 2007;115:654-7.

16. Verheyen N, Fahrleitner-Pammer A, Belyavskiy E, Gruebler MR, Dimai HP, Amrein K, et al. Relationship between bone turnover and left ventricular function in primary hyperparathyroidism: The EPATH trial. PLoS ONE. 2017;12:e0173799.

17. Bozic B, Loncar G, Prodanovic N, Radojicic Z, Cvorovic V, Dimkovic S, et al. Relationship between high circulating adiponectin with bone mineral density and bone metabolism in elderly males with chronic heart failure. J Card Fail. 2010;16:301-7.

18. Wu C, Kato TS, Pronschinske K, Qiu S, Naka Y, Takayama H, et al. Dynamics of bone turnover markers in patients with heart failure and following haemodynamic improvement through ventricular assist device implantation. Eur J Heart Fail. 2012;14:1356-65.

19. Yeap BB, Chubb SAP, Flicker L, McCaul KA, Ebeling PR, Hankey GJ, et al Associations of total osteocalcin with all-cause and cardiovascular mortality in older men. The Health In Men Study. Osteoporos Int. 2012;23:599-606.

20. Yamashita T, Okano K, Tsuruta Y, Akiba T, Nitta K. Serum osteocalcin levels are useful as a predictor of cardiovascular events in maintenance hemodialysis patients. Int Urol Nephrol. 2013;45:207-14.

21. Hwang YC, Kang M, Cho IJ, Jeong IK, Ahn KJ, Chung HY, et al. Association between the circulating total osteocalcin levels and the development of cardiovascular disease in middle-aged men: a mean 8.7-year longitudinal followup study. J Atheroscler Thromb. 2015;22:136-43.

22. Bao YQ, Ma XJ, Yang R, Wang FF, Hao YP, Dou JX, et al. Inverse relationship between serum osteocalcin levels and visceral fat area in Chinese men. J Clin Endocrinol Metab. 2013;98:345-51.

23. Luo YQ, Ma XJ, Hao YP, Pan XP, Xu YT, Xiong $Q$, et al. Inverse relationship between serum osteocalcin levels and nonalcoholic fatty liver disease in postmenopausal Chinese women with normal blood glucose levels. Acta Pharmacol Sin. 2015;36:1497-502.

24. Kareinen A, Viitanen L, Halonen P, Lehto S, Laakso M. Cardiovascular risk factors associated with insulin resistance cluster in families with early-onset coronary heart disease. Arterioscler Thromb Vasc Biol. 2001;21:1346-52.

25. Pi M, Quarles LD. Multiligand specificity and wide tissue expression of GPRC6A reveals new endocrine networks. Endocrinology. 2012;153:2062-9.

26. Wellendorph $P$, Bräuner-Osborne $H$. Molecular cloning, expression, and sequence analysis of GPRC6A, a novel family C G-protein-coupled receptor. Gene. 2004;335:37-46

27. Gamal SM, Sadek NB, Rashed LA, Shawky HM, Gamal EM. Effect of gammacarboxylase inhibition on serum osteocalcin may be partially protective against developing diabetic cardiomyopathy in type 2 diabetic rats. Diab Vasc Dis Res. 2016;13:405-17.

28. Jung $\mathrm{CH}$, Lee WJ, Hwang JY, Lee MJ, Seol SM, Kim YM, et al. The preventive effect of uncarboxylated osteocalcin against free fatty acid-induced endothelial apoptosis through the activation of phosphatidylinositol 3-kinase/Akt signaling pathway. Metabolism. 2013;62:1250-7.

29. Luo YQ, Ma XJ, Hao YP, Xiong Q, Xu YT, Pan XP, et al. Relationship between serum osteocalcin levels and carotid intima-media thickness in a metabolically healthy Chinese population. Cardiovasc Diabetol. 2015;14:82.

30. Jung KY, Kim KM, Ku EJ, Kim YJ, Lee DH, Choi SH, et al. Age- and sex-specific association of circulating osteocalcin with dynamic measures of glucose homeostasis. Osteoporos Int. 2016;27:1021-29.

31. Jagtap VR, Ganu JV, Nagane NS. BMD and serum intact osteocalcin in postmenopausal osteoporosis women. Indian J Clin Biochem. 2011;26:70-3. 